\title{
Comparative study for non-statistical fluctuation of net- proton, baryon, and charge multiplicities
}

\author{
Dai-Mei Zhou ${ }^{1}{ }^{*}$, Zeng-Zeng Luo ${ }^{1}$, Yun cheng ${ }^{1}$, Ayut Limphirat ${ }^{2,3}$, \\ Yu-Liang Yan ${ }^{4}$, Yu-Peng $\mathrm{Yan}^{2,3}, \mathrm{Xu} \mathrm{Cai}^{1}$, and Ben-hao Sa ${ }^{1,4}$ \\ ${ }^{1}$ Key Laboratory of Quark and Lepton Physics (MOE) and Institute of Particle Physics, \\ Central China Normal University, Wuhan 430079, China. \\ ${ }^{2}$ School of Physics, Institute of Science, Suranaree University of Technology, Nakhon Ratchasima 30000, Thailand. \\ 3 Thailand Center of Excellence in Physics (ThEP), \\ Commission on Higher Education, Bangkok 10400, Thailand. \\ ${ }^{4}$ China Institute of Atomic Energy, P. O. Box 275 (10), Beijing, 102413 China.
}

\begin{abstract}
We calculate the real and non-statistical higher moment excitation functions $\left(\sqrt{s_{N N}}=11.5\right.$ to $200 \mathrm{GeV}$ ) for the net-proton, net-baryon, and the net-charge number event distributions in the relativistic $\mathrm{Au}+\mathrm{Au}$ collisions with the parton and hadron cascade model PACIAE. It turned out that because of the statistical fluctuation dominance it is very hard to see signature of the CP singularity in the real higher moment excitation functions. It is found that the property of higher moment excitation functions are significantly dependent on the window size, and hence the CP signatures may show only in a definite window for a given conserved observable non-statistical higher moments. But for a given widow size, the CP singularity may show only in the non-statistical higher moment excitation functions of a definite conserved observable.
\end{abstract}

PACS numbers: 25.75.Dw, 24.85.+p

\section{INTRODUCTION}

One fundamental aim of relativistic heavy-ion collisions is to explore the phase transition from the hadronic matter (HM) to the quark-gluon matter (QGM). Evidences for the strongly coupled quark-gluon plasma (sQGP) were reported years ago [1-4]. The RHIC beam energy scan (BES) was then proposed [5]. Attempts are being made to experimentally locate the QCD critical point $(\mathrm{CP})$, where the first order phase transition gives over to the "crossover" [6] in the QCD phase diagram of temperature $T$ vs. baryon chemical potential $\mu_{B}\left[\begin{array}{l}7 \\ -10\end{array}\right]$. Because a cms energy $\left(\sqrt{s_{N N}}\right)$ in the heavy-ion collisions maps out a $\mu_{B}$ value, a $T$ value can be extracted by fitting the particle ratio in the hadronic resonance gas (HRG) model [11, 12] to corresponding data in the heavy-ion collisions. The scanning over the phase diagram can then be performed. Meanwhile, the search for CP signatures could also be done at each cms energy via the singularity (nonmonotonicity) shown in higher moment excitation functions (higher moment as a function of reaction energy) of the conserved observables. Since the birth of RHIC BES considerable progresses have been made in this field both experimentally [13-16] and theoretically [17 24]. A upsurge was reached last year at the $7^{\text {th }}$ International Workshop on Critical Point and Onset of Deconfinement, Nov. 7-11 Wuhan, China. Although many relevant fundamental physics have been demonstrated, the mysteries remain.

Early in 2009 one employed the UrQMD model in-

\footnotetext{
*zhoudm@phy.ccnu.edu.cn/zdm@iopp.ccnu.edu.cn
}

vestigating the net-proton, net-baryon, and net-charge kurtosis in the central $\mathrm{Pb}+\mathrm{Pb} / \mathrm{Au}+\mathrm{Au}$ collisions from $E_{l a b}=2 A \mathrm{GeV}$ to $\sqrt{s_{N N}}=200 \mathrm{GeV}[25]$. They did not find any evidence of $\mathrm{CP}$ singularity in the kurtosis excitation functions. However, they did find that the kurtosis excitation functions behave quite different among the net-proton, net-baryon, and net-charge: The net-proton kurtosis becomes slightly negative at low $\sqrt{s_{N N}}$ and the net-baryon kurtosis decreases to a large negative value while the net-charge kurtosis keeps zero approximately. Similarly, the kurtosis as a function of the rapidity window size were also different: Both the net-proton kurtosis and net-charge kurtosis are differently fluctuated around zero, but the net-baryon kurtosis reaches a large negative value at the lager window. On the contrary, the opinion in [26] is quite opposite. By assuming that the number distribution of a conserved observable is a binomial function and the strange baryon can be approximately considered, they analytically derived a relation between baryon number cumulant and the proton number cumulant. This means that the net-baryon cumulant has a similar behavior to the net-proton.

Recently it has been reported in [27] that the effect of finite acceptance dramatically influences the cumulants of conserved observables, which makes the interpretation of experimental data measured in a given acceptance very uncertain. In [27] the concept of "required acceptance" is introduced, where all the particles, which are necessary for the physics in question, are covered. The required acceptance is not the full acceptance where all particles in the final hadronic state are covered. The authors assume that the bias of a real acceptance in a real experiment from the required acceptance can be represented by a binomial probability distribution, then they derive the 
general formula that relate the true cumulants which reflect the full dynamics of the system (require acceptance) with the actually measured cumulants (real acceptance). This relation involves additional moments which cannot be expressed by the cumulants and should be simultaneously measured in order to extract information about $\mathrm{CP}$ singularity. But how to measure these additional moments is not yet known.

We attempt, in this paper, to further explore the relevant issues using the parton and hadron cascade model PACIAE [28]. The net-proton, net-baryon, and the netcharge number event distributions and their higher moment excitation functions $\left(\sqrt{s_{N N}}=11.5\right.$ to $\left.200 \mathrm{GeV}\right)$ in the $0-5 \%$ most central $\mathrm{Au}+\mathrm{Au}$ collision are calculated in different transverse momentum and pseudorapidity windows by the PACIAE model. As pointed out in 29] that the study of higher moment excitation function singularity is a matter of dynamical fluctuation, but the statistical fluctuation is always dominant. In order to pronounce the effect of dynamical fluctuation, non-statistical higher moments (cumulants) of conserved observables are proposed in [29]. Therefore, we calculate simultaneously both the real and the non-statistical higher moment excitation functions, and comparatively analyze the dependence of these excitation functions on the window size.

\section{MODELS}

Suppose that the event distribution of the number of conserved observable $x$ is $P(x)$, then its $\mathrm{n}^{\text {th }}$ moment about zero is

$$
\left\langle x^{(n)}\right\rangle=\int x^{n} P(x) d x
$$

and its $\mathrm{n}^{\text {th }}$ moment about the mean $\left(M \equiv\left\langle x^{(1)}\right\rangle \equiv\langle x\rangle\right)$ is

$$
M^{(n)}=\left\langle(x-\langle x\rangle)^{n}\right\rangle=\int(x-\langle x\rangle)^{n} P(x) d x .
$$

Therefore its higher moments and moment products investigated widely are:

$$
\begin{gathered}
\text { variance : } \quad \sigma^{2}=M^{(2)}, \\
\text { skewness : } \quad S=M^{(3)} /\left(M^{(2)}\right)^{3 / 2}, \\
\text { kurtosis : } \quad \kappa=M^{(4)} /\left(M^{(2)}\right)^{2}-3, \\
S \sigma=M^{(3)} / M^{(2)},
\end{gathered}
$$

and

$$
\kappa \sigma^{2}=M^{(4)} / M^{(2)}-3 M^{(2)} .
$$

We employ the parton and hadron cascade model PACIAE to generate the real event distributions. The mixed events are generated by combining particles selected randomly from different real events, while reproducing the event multiplicity distribution of the real events [30]. If the $\mathrm{n}^{\text {th }}$ real (mixed) moment about the mean calculated with real (mixed) events is denoted by $M_{R}^{(n)}\left(M_{M}^{(n)}\right)$, then the corresponding $\mathrm{n}^{\text {th }}$ non-statistical moment is

$$
M_{N O N}^{(n)}=M_{R}^{(n)}-M_{M}^{(n)},
$$

like in 30 .

PACIAE is based on PYTHIA 31] which is devised for the high energy hadron-hadron (hh) collisions. In the PYTHIA model, a hh collision is first decomposed into the parton-parton collisions. The hard parton-parton interaction is dealt with lowest leading order perturbative QCD (LO-pQCD) and the soft parton-parton interaction is considered empirically. Because the consideration of the initial- and final- state parton showers as well as the parton multiple interactions, the consequence of a $\mathrm{hh}$ collision is a partonic state composed of quarks (antiquarks), diquarks (anti-diquarks) and gluons. It is followed by the string construction and fragmentation. So a final hadronic state is obtained for a hh collision eventually.

In the PACIAE model [28], a nucleus-nucleus collision is first decomposed into nucleon-nucleon (NN) collisions according to the collision geometry and NN total cross section. Each NN collision is dealt by PYTHIA with string fragmentation switched-off and the diquarks (antidiquarks) broken into quark pairs (anti-quark pairs). When all NN collision pairs are exhausted, a partonic initial state (composed of quarks, antiquarks, and gluons) is obtained for a nucleus-nucleus collision. This partonic initial stage is followed by a parton evolution stage, where parton rescattering is performed by the Monte Carlo method with $2 \rightarrow 2$ LO-pQCD cross sections 32]. The hadronization stage follows the parton rescattering stage. The Lund string fragmentation model and a phenomenological coalescence model are provided for the hadronization. Then the rescattering among the produced hadrons is dealt with the usual two body collision model [28]. In this hadronic evolution stage, only the rescatterings among $\pi, K, p, n, \rho(\omega), \Delta, \Lambda, \Sigma, \Xi, \Omega$, and their antiparticles are considered for simplicity. We refer to [28] for the details.

\section{RESULTS}

We use PACIAE to generate the net-proton, netbaryon and the net-charge number event distributions in $0-5 \%$ most central $\mathrm{Au}+\mathrm{Au}$ collisions at $\sqrt{s_{N N}}=11.5$ to $200 \mathrm{GeV}$ for the different acceptance sizes. The real and non-statistical higher moment excitation functions are then calculated, with all model parameters fixed. Baryons are identified with $p+n+\Lambda$ and their antiparticles (the non-strange baryons heavier than neutron and the strange baryons heavier than $\Lambda$ are assumed decay already) and the charge with $\pi^{ \pm}, K^{ \pm}, p$ and $\bar{p}$. 

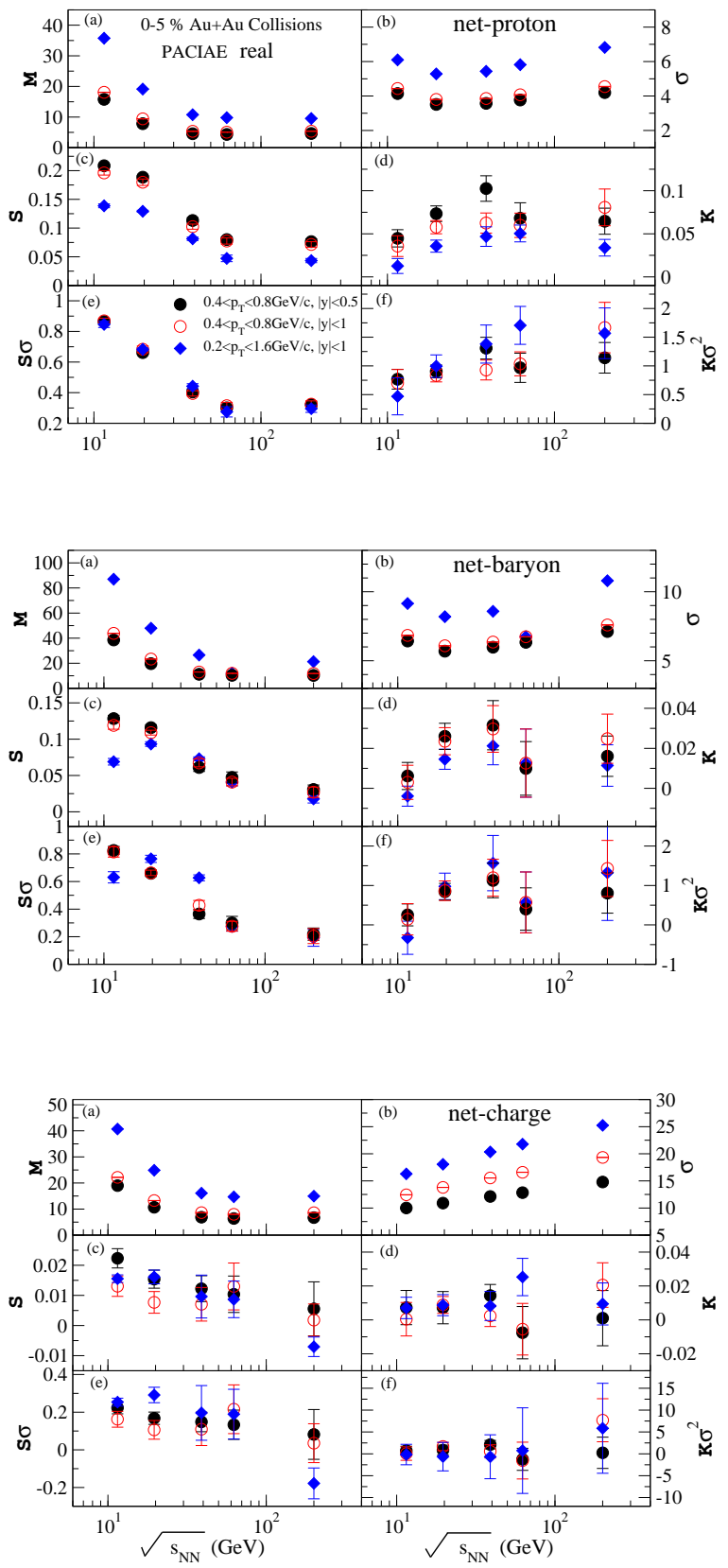

FIG. 1: (color online) Real higher moment excitation functions of the net-proton (top), net-baryon (middle), and the net-charge (bottom) in the $0-5 \%$ most central $\mathrm{Au}+\mathrm{Au}$ collisions calculated by PACIAE for different window sizes.

We summarize our results as follows:

Shown in the Figure 1, from the top to the bottom, are respectively the net-proton, net-baryon, and the netcharge real higher moment excitation functions, with the mean $M$, the variance $\sigma$, skewness $S$, kurtosis $\kappa$, and the products $S \sigma$ and $\kappa \sigma^{2}$ displayed in the panels (a), (b), (c), (d), (e) and (f), respectively. In the figure, the solid circles are the results in the acceptances of $0.4<p_{T}<0.8$ $\mathrm{GeV} / \mathrm{c}$ and $|y|<0.5$, the open circles are the results in $0.4<p_{T}<0.8$ and $|y|<1$, and the diamonds are the

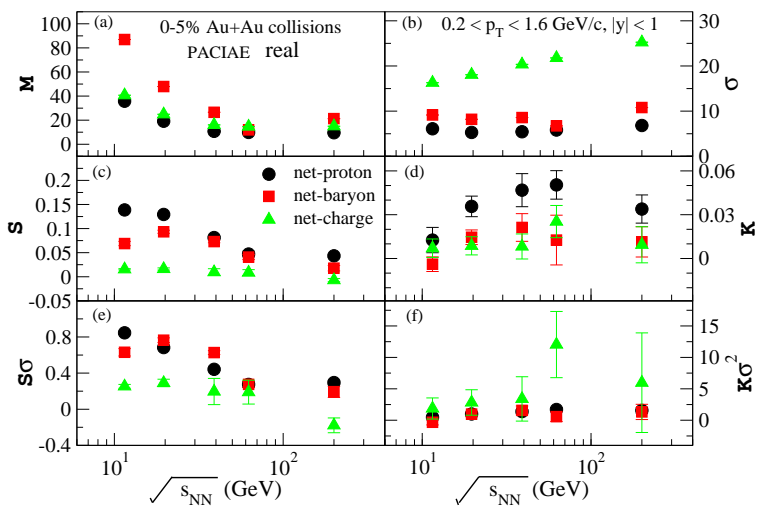

FIG. 2: (color online) Real higher moment excitation functions of the net-proton, net-baryon, and the net-charge in the $0-5 \%$ most central $\mathrm{Au}+\mathrm{Au}$ collisions calculated by PACIAE for a given window size of $0.2<p_{T}<1.6 \mathrm{GeV} / \mathrm{c}$ and $|y|<1$.

results in $0.2<p_{T}<1.6$ and $|y|<1$.

Figure 2 gives, respectively, the net-proton (circles), net-baryon (squares), and the net-charge (triangles) real higher moment excitation functions for the mean (panel (a)), $\sigma((\mathrm{b}))$, skewness $((\mathrm{c}))$, kurtosis $((\mathrm{d})), S \sigma((\mathrm{e}))$, and $\kappa \sigma^{2}((\mathrm{f}))$ calculated in the $0.2<p_{T}<1.6 \mathrm{GeV} / \mathrm{c}$ and $|y|<1$ acceptances.

We see from these figures that:

- The shape of net-proton (net-baryon, net-charge) real higher moment excitation function changing with the window sizes is approximately similar only for the mean and $\sigma$, see top panel (a) and (b) (middle panel (a) and (b), as well as bottom panel (a) and (b)) of Fig. 1. This change becomes rather complicated for the skewness $S$, kurtosis $\kappa$, and the product $S \sigma$ and $\kappa \sigma^{2}$.

- We see in Fig. 1 that the net-proton kurtosis is always positive (cf. top panel (d)) unlike in [25], the net-baryon kurtosis decreases to a small negative value at low $\sqrt{s_{N N}}$ (cf. middle panel (d)) but not to a lager negative value as mentioned in 25], while the net-charge kurtosis approximately keeps zero (cf. bottom panel (d)) like in [25].

- It is hard to see any signature, which may indicate the CP singularity, from the real higher moment excitation functions shown in these figures. Only the net-charge real excitation function of kurtosis in the window of $0.4<p_{T}<0.8 \mathrm{GeV} / \mathrm{c}$ and $|y|<0.5$ as well as $0.4<p_{T}<0.8 \mathrm{GeV} / \mathrm{c}$ and $|y|<1$ may indicate some signature (cf. bottom panel (d) in Fig. (1).

Figure 3 gives, from the top to the bottom, the netproton, net-baryon, and the net-charge non-statistical higher moment excitation functions for the skewness (panel (a)), kurtosis (panel (b)), $S \sigma$ (panel (c)), and $\kappa \sigma^{2}$ (panel (d)). In the figure the solid circles, open circles, and the diamonds are, respectively, the results in the 

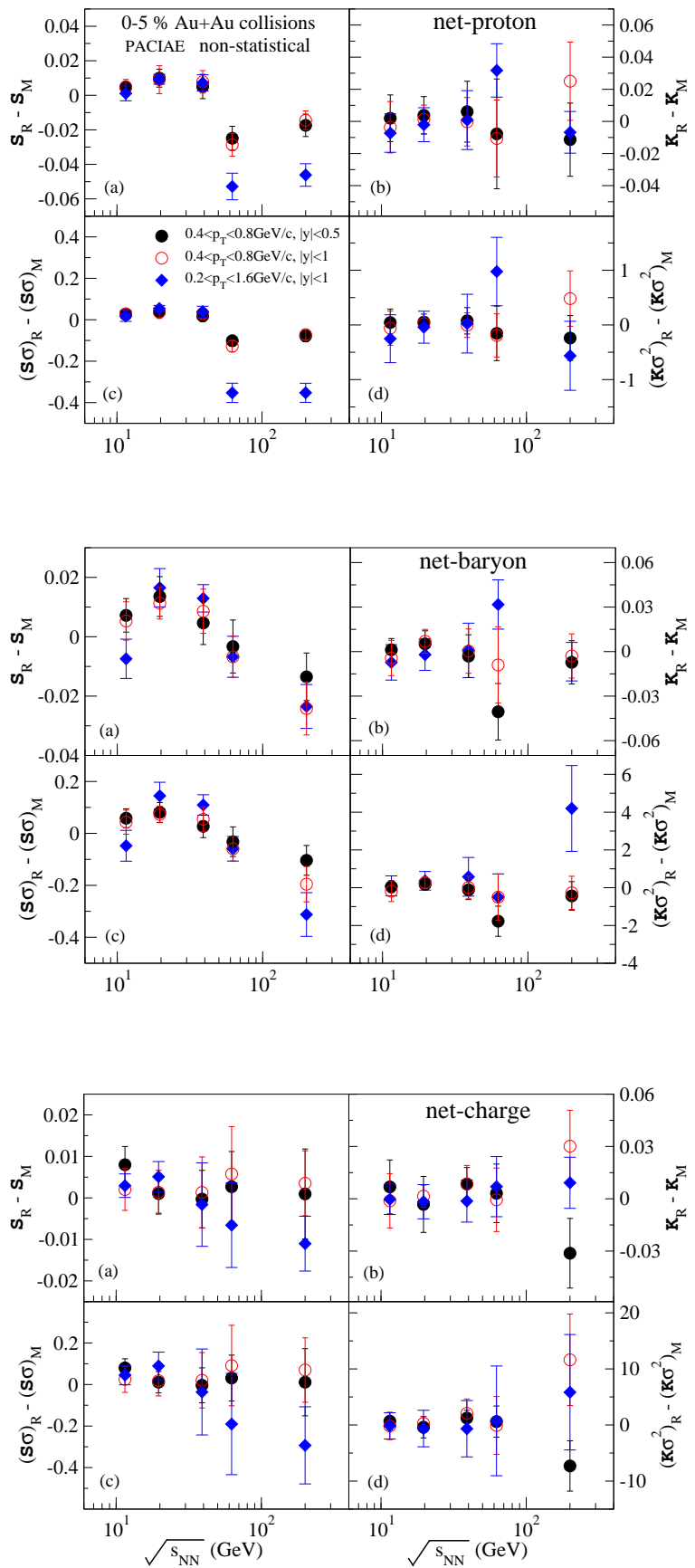

FIG. 3: (color online) Non-statistical higher moment excitation functions of the net-proton (top), net-baryon (middle), and the net-charge (bottom) in the $0-5 \%$ most central $\mathrm{Au}+\mathrm{Au}$ collisions calculated by PACIAE for different window sizes.

acceptances of $0.4<p_{T}<0.8 \mathrm{GeV} / \mathrm{c}$ and $|y|<0.5$, $0.4<p_{T}<0.8$ and $|y|<1$, and $0.2<p_{T}<1.6$ and $|y|<1$. One sees an interesting structure, as shown in the net-proton non-statistical excitation functions of the skewness and $S \sigma$ (cf. top panels (a) and (c) in Fig. 3), which becomes more pronounced with increasing the window size. The structure that the net-proton skewness and $S \sigma$ go down to a sizably negative value within a small

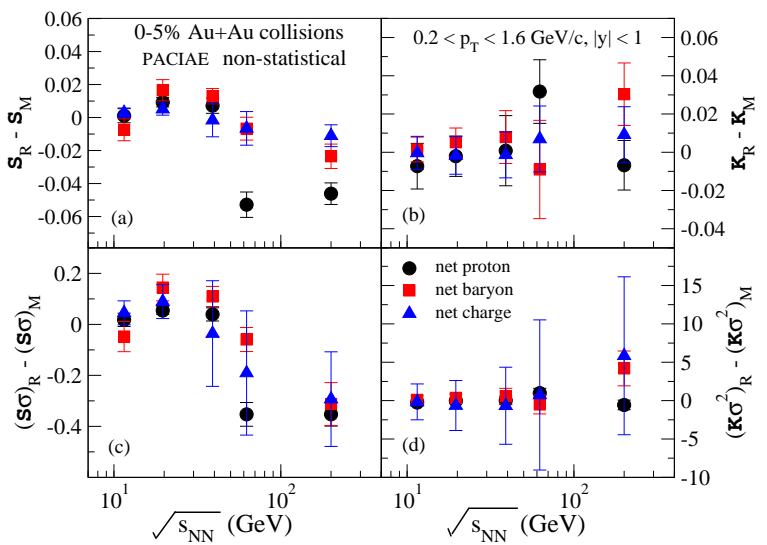

FIG. 4: (color online) Non-statistical higher moment excitation functions of the net-proton (top), net-baryon (middle), and the net-charge (bottom) in the $0-5 \%$ most central $\mathrm{Au}+\mathrm{Au}$ collisions calculated by PACIAE for a given window sizes of $0.2<p_{T}<1.6 \mathrm{GeV} / \mathrm{c}$ and $|y|<1$.

energy region may serve as a signature of the $\mathrm{CP}$ singularity. As for the net-baryon non-statistical excitation functions, it is the kurtosis and $\kappa \sigma^{2}$ which may display the CP singularity only in the window of $0.4<p_{T}<0.8$ $\mathrm{GeV} / \mathrm{c}$ and $|y|<0.5$, as shown in the middle panels (b) and (d). However, one can not find any possible signature of the CP singularity in the bottom panels.

Figure 4 gives, respectively, the net-proton (circles), net-baryon (squares), and the net-charge (triangles) nonstatistical excitation functions for the skewness (panel (a)), kurtosis (panel (b)), $S \sigma$ (panel (c)), and $\kappa \sigma^{2}$ (panel (d)) calculated in the acceptance of $0.2<p_{T}<1.6$ $\mathrm{GeV} / \mathrm{c}$ and $|y|<1$. We see in Fig 4 (a) and (c) that the $\mathrm{CP}$ singularity may be shown in the non-statistical excitation functions for the net-proton but not for the netbaryon and net-charge. However, a CP signature may appear in the non-statistical excitation functions of kurtosis for the net-baryon but not for the net-proton and net-charge, as shown in Fig团(b).

\section{CONCLUSIONS}

We have calculated the real and non-statistical higher moment excitation functions $\left(\sqrt{s_{N N}}=11.5\right.$ to $\left.200 \mathrm{GeV}\right)$ for the net-proton, net-baryon, and the net-charge number event distributions in the relativistic $\mathrm{Au}+\mathrm{Au}$ collisions with the parton and hadron cascade model PACIAE [28]. The real event is generated with the PACIAE model, while the mixed event is constructed by combining particles selected from the real events randomly. The non-statistical moment is derived as the difference between real moment and the mixed moment. It turned out that because of the statistical fluctuation dominance [29] it is very hard to see any signature of the CP singularity in the real higher moment excitation functions of the net-proton, net-baryon, and the net-charge, which is 
consistent with the results from the real higher moment studies in 13, 25].

The work suggests that the non-statistical moment of conserved observables may play a potential role in exploring the $\mathrm{CP}$ singularity in the relativistic heavy ion collisions. However, it is found that the property of higher moment excitation functions is significantly dependent on the window size. For a given conserved observable, a signature of the CP singularity may appear in its non-statistical higher moment excitation functions only in a definite window size. But for a given widow size, the CP singularity may be shown only in the non- statistical higher moment excitation functions of a definite conserved observable as the multiplicity distribution over rapidity and transverse momentum among different conserved observables can be rather different.

Acknowledgements: This work was supported by the National Natural Science Foundation of China under grant nos.: 10975062, 11075217, 11105227, 11175070, 11221504,11205066 and by the 111 project of the foreign expert bureau of China. AL and YPY acknowledge the financial support from TRF-CHE-SUT under contract No. MRG5480186.
[1] I. Arsene, et al., BRAHMS Collaboration, Nucl. Phys. A 757, 1 (2005).

[2] B. B. Back, et al., PHOBOS Collaboration, Nucl. Phys. A 757, 28 (2005).

[3] J. Admas, et al., STAR Collaboration, Nucl. Phys. A 757, 102 (2005).

[4] K. Adcox, et al., PHENIX Collaboration, Nucl. Phys. A 757, 184 (2005).

[5] STAR Internal note SN0493, 2009; B. Mohanty, Nucl. Phys. A 830, 899c (2009); B. I. Abelev et al., Phys. Rev. C 81, 024911 (2010).

[6] Y. Aoki et al., Nature (London) 443, 675 (2006).

[7] Y. aoki et al., Phys. Lett. B 643, 46 (2006).

[8] M. Cheng, et al., Phys. Rev. D 74, 054507 (2006).

[9] Z. Fodor and S. D. Katz, JHEP 04, 050 (2004).

[10] R. V. Gavai and S. Gupta, Phys. Rev. D 78, 114503 (2008).

[11] P. Braun-Munzinger, J. Stachel, J. P. Wessels, and N. Xu, Phys. Lett. B 365, 1 (1996).

[12] J. Cleymans, D. Elliott, H. Satz, and R. L. Thews, Z. Phys. C 74, 319 (1997).

[13] M. Aggarwal, et al., STAR Collaboration, Phys. Rev. Lett. 105, 022302 (2010).

[14] S. Gupta, Xiaofeng Luo, B. Mohanty, H. G. Ritter, X. $\mathrm{Nu}$, Science 332, 1525 (2011).

[15] Xiaofeng Luo for STAR Collaboration, J. Phys.: Conf. Ser. 316, 012003 (2011) and Acta Physica Polonica B Proceedings Supplement 5, 497 (2012).

[16] B. Mohanty, J. Phys. G: Nucl. Part. Phys. 38, 124023, (2011).

[17] F. Karsch and K. Redlich, arXiv: 1107.1412v1; R. V. Gavai, Proceedings of Strangeness in Quark Matter, Krakow, September 18-24, 2011, to appear in Acta Physica Polonica B.

[18] B.-J. Schaefer and M. Wagner, Phys. Rev. D 85, 034027,
(2012).

[19] P. Costa, C. A. De Sousa, and M. C. Ruivo, arXiv: 1112.6306v1.

[20] Si-Xue Qin, Lei Chang, Huan Chen, Yu-Xin Liu and C. D. Roberts, Phys. Rev. Lett. 106, 172301 (2011).

[21] C. B. Yang and Xin Wang, Phys. Rev. C 84, 064908 (2011).

[22] P. Braun-Munzinger, B. Friman, F. Karsch, K. Redlich, and V. Skokov, Nucl. Phys. A 880, 48 (2012).

[23] Lizhu Chen, Xue Pan, Fengbo Xiong, Lin Li, Na Li, Zhiming Li, Gang Wang, and Yufang Wu, J. Phys. G: Nucl. Part. Phys. 38, 115004, (2011).

[24] D. J. Wang, L. P. Csernai, D. Strottman, Cs. Anderlik, Y. Cheng, D. M. Zhou, Y. L. Yan, X. Cai and B. H. Sa, arXiv:1205.4934 1 [nucl-th].

[25] M. Nahrgang, T. Schuster, R. Stock, M. Mitrovski, and M. Bleicher, arXiv:0903.2911 [hep-ph].

[26] M. Kitazawa and M. Asakawa, Phys. Rev. C 86, 024904 (2012), arxiV: 1205.3292v1[nucl-th].

[27] A. Bzdak and V. Koch, Phys. Rev. C 86, 044904 (2012).

[28] Ben-Hao Sa, Dai-Mei Zhou, Yu-Liang Yan, Xiao-Mei Li, Sheng-Qin Feng, Bao-Guo Dong, and Xu Cai, Comput. Phys. Comm. 183, 333 (2012).

[29] Dai-Mei Zhou, Ayut Limphirat, Yu-liang Yan, Cheng Yun, Yu-peng Yan, Xu Cai, Laszlo P. Csernai, and BenHao Sa, Phys. Rev. C 85, 064916 (2012).

[30] S. V. Afanasiev, et al., NA49 Collaboration, Phys. Rev. Lett. 86, 1965 (2001); C. Alt, et al., NA49 Collaboration, Phys. Rev. C 79, 044910 (2009); T. Anticic, et al., NA49 Collaboration, Phys. Rev. C 83, 061902 (2011).

[31] T. Sjöstrand, S. Mrenna, and P. Skands, JHEP 05, 026 (2006).

[32] B. L. Combridge, J. Kripfgang, and J. Ranft, Phys. Lett. B 70, 234 (1977). 